\title{
Diagnosis de una patología en gres porcelánico pegado en fachada
}

\section{Diagnosis of stoneware pathology placed on bonded façade}

\author{
$\underline{\text { L. Delgado-Méndez }}{ }^{(*)}$, C. del Moral-Ávila ${ }^{(*)}$, I. Valverde-Palacios ${ }^{(*)}$ I. Valverde-Espinosa ${ }^{(*)}$
}

\section{RESUMEN}

Esta investigación analiza las causas del desprendimiento de un revestimiento de gres porcelánico colocado en fachada. En el momento de abordar este trabajo comprobamos que hay métodos normalizados para la determinación de las características de los adhesivos para las baldosas cerámicas y de las propias baldosas, pero en ambos casos, son métodos a aplicar durante el proceso de fabricación. No existen métodos normalizados para la determinación de las características de aplacados en servicio, cuestión que aborda este trabajo. Las técnicas de diagnosis empleadas han consistido en la comprobación de la capacidad de adherencia del adhesivo, la localización de juntas de movimiento, el análisis de las juntas de colocación y la detección de anclajes mecánicos ocultos.

Finalmente, los resultados obtenidos tras la aplicación de dicha metodología para el análisis de la patología han permitido establecer las causas que han originado los daños, proponiéndose como método de diagnosis del desprendimiento de un aplacado cerámico en servicio.

Palabras clave: Porcelánico; fachadas; adhesivos cementosos; adherencia.

\section{ABSTRACT}

This research examines the causes of the detachment of stoneware placed on façade cladding. In dealing with this work we see that there are standard methods for the determination of the characteristics of adhesives for ceramic tiles and own tiles, but in both cases, the methods are to be applied during the manufacturing process. There are no standard methods for the determination of the characteristics of facings in service, which deals with this work. Employed diagnosis techniques have consisted of checking the capacity of adhesion of adhesive, the location of movement joints, joints of positioning analysis and detection of hidden mechanical anchors.

Finally, the results obtained after the application of this methodology to the analysis of Pathology have allowed establishing the causes that have given rise to the damage; we propose a method of diagnosis of the evolution of a ceramic covering.

Keywords: Porcelain; façades; cementitious adhesives; adhesion.

(*) Universidad de Granada (España).

Persona de contacto/Corresponding author: ldelgado@ugr.es (L. Delgado-Méndez)

Cómo citar este artículo/Citation: Delgado-Méndez, L., del Moral-Ávila, C., Valverde-Palacios, I., Valverde-Espinosa, I. (2015). Diagnosis de una patología en gres porcelánico pegado en fachada. Informes de la Construcción, 67(539): e108, doi: http://dx.doi. org/10.3989/ic.14.085.

Licencia / License: Salvo indicación contraria, todos los contenidos de la edición electrónica de Informes de la Construcción se distribuyen bajo una licencia de uso y distribución Creative Commons Reconocimiento no Comercial 3.o. España (cc-by-nc). 


\section{INTRODUCCIÓN}

Esta investigación analiza las causas del desprendimiento de un revestimiento de gres porcelánico colocado en fachada.

En el momento de abordar este trabajo comprobamos que hay métodos normalizados para la determinación de las características de los adhesivos para las baldosas cerámicas, como la Norma sobre Adhesivos para baldosas cerámicas. Requisitos, evaluación de la conformidad, clasificación y designación (1), la de Adhesivos para baldosas cerámicas. Determinación de la resistencia a la tracción de los adhesivos cementosos (2) o la de Adhesivos para baldosas cerámicas. Determinación de la deformación transversal de adhesivos y materiales de rejuntado cementosos (3) y de las propias baldosas, pero en todos los casos, son métodos a aplicar durante el proceso de fabricación.

No existen métodos normalizados para la determinación de las características de aplacados en servicio, cuestión que aborda este trabajo como consecuencia de los daños por desprendimientos de las baldosas que se estaban produciendo en las fachadas de una promoción de viviendas de reciente construcción.

\section{DESCRIPCIÓN DEL EDIFICIO, SISTEMAS CONSTRUCTIVOS Y DAÑOS}

\subsection{Descripción del edificio y sistemas constructivos}

La urbanización donde se producen los daños en las fachadas se encuentra situada en la provincia de Granada y está compuesta por varios bloques aislados de viviendas plurifamiliares, de 4 a 6 plantas de altura, rodeados de espacios libres de uso privativo.

Las fachadas de los bloques se han ejecutado con un cerramiento compuesto por una citara de ladrillo, cámara de aire y trasdosado autoportante interior de placas de yeso laminado con aislamiento térmico. La citara de ladrillo exterior se ha terminado con un revestimiento de baldosas de gres porcelánico pegado al soporte. Se han utilizado dos tipos de baldosas de gran formato: $66 \times 37 \mathrm{~cm}$ y $50 \times 33 \mathrm{~cm}$. En total se ejecutó una superficie aproximada de $13.000 \mathrm{~m}^{2}$ de aplacado cerámico.

El proyecto se redactó en 2005, terminándose los bloques entre 2007 y 2008, según la fase. El encargo de esta investigación procede de la comunidad de propietarios, tras varios intentos fallidos de reparación llevados a cabo por el promotor y el constructor.

Se interpuso una demanda judicial y a finales de 2013 se llegó a un acuerdo extrajudicial de indemnización antes de celebrase el juicio.

\subsection{Daños}

El daño principal en el revestimiento exterior consiste en el despegue total o parcial de las baldosas, por abombamiento en diferentes zonas, que acaban desprendiéndose. La caída de las baldosas se produce de forma dispersa en todos los bloques, no siendo posible acceder a los datos necesarios para establecer una relación entre las zonas donde se desprenden y posibles circunstancias de ubicación que propicien estos desprendimientos (repetición de caídas en las mismas zonas de los distintos bloques, orientaciones, etc.).

También se observan manchas en forma de chorreones blanquecinos que se originan en las juntas horizontales de mortero entre baldosas.

\section{METODOLOGÍA}

En primer lugar se comprobó que las especificaciones del Proyecto eran adecuadas al sistema constructivo elegido, con el fin de descartar un error de proyecto como causa de los daños en las fachadas.

En el Proyecto se especificaba un aplacado de baldosas de gres porcelánico enmalladas por su trasdós, recibidas con cemento cola de ligantes mixtos, tipo $\mathrm{C} 2$ (designación conforme con la establecida por la UNE-EN Adhesivos para baldosas cerámicas. Requisitos, evaluación de la conformidad, clasificación y designación (1), para los adhesivos cementosos mejorados, a los que se le exige una adherencia $\geq 1 \mathrm{~N} / \mathrm{mm}^{2}$ ), sobre enfoscado maestreado del paramento y fijadas con anclajes invisibles de retención de acero inoxidable. Estas previsiones de Proyecto son adecuadas para el sistema constructivo elegido, descartándose el error en las especificaciones del mismo como la causa de los daños.

En segundo lugar se procedió a diagnosticar los citados daños en base a las siguientes comprobaciones de campo:

a) Determinación de los parámetros técnicos de referencia.

b) Comprobación de la capacidad de adherencia y del método de aplicación del material de agarre.

c) Comprobación de la existencia de los anclajes mecánicos de las baldosas.

d) Análisis de las juntas de colocación entre baldosas.

e) Análisis de las juntas de movimiento del revestimiento.

\subsection{Determinación de los parámetros técnicos de referencia}

El gres porcelánico es un material derivado de la arcilla cocida, y pertenece al grupo de lo que podría llamarse «baldosas cerámicas de última generación». Es el tipo de baldosas cerámicas de más reciente aparición en el mercado en España, siendo el fruto de una alta tecnología en su proceso de fabricación, con una cuidadosa selección de las materias primas, sometimiento a unas fuertes presiones por prensado en la conformación de las piezas y cocción a más altas temperaturas que cualquiera de las otras piezas cerámicas que utilizamos en construcción, como azulejos y gres (4).

Fruto de este proceso de fabricación son unas piezas con unas propiedades características, de las que caben destacar su alta resistencia mecánica y su bajísima capacidad de absorción de agua, inferior al o,5 \%, llegando en algunos casos hasta el $0,1 \%$ (4). Esta baja absorción de agua hace que se requieran de técnicas especiales para su correcta colocación.

Aunque comparte técnicas de colocación con otros materiales constituidos por piezas rígidas planas, tipo baldosas, como la disposición de juntas entre piezas, de juntas de dilatación y de anclajes mecánicos de seguridad, otras técnicas son específicas por las singularidades del material, como la utilización de adhesivos especiales. 
Para el análisis de las fachadas que nos ocupan hemos partido de los siguientes parámetros técnicos de referencia.

\subsubsection{Adherencia de los materiales de revestimiento de las fachadas}

El gres porcelánico en fachadas no puede colocarse con un mortero de cemento tradicional. La adherencia entre las baldosas y el mortero tradicional se consigue porque el mortero de cemento penetra en los poros de la baldosa, y al solidificar, queda mecánicamente unido a ella (4). El gres porcelánico no es un material poroso, perteneciendo al Grupo I de la clasificación de las baldosas cerámicas establecida por la Norma UNE-EN 14411 (5) según su absorción de agua, al presentar una absorción de agua prácticamente nula, $<0,5 \%$, por lo que hay que emplear productos con un alto poder de adherencia química. Como además las baldosas están en el exterior, sometidas a fuertes cambios de temperatura y por lo tanto a dilataciones y contracciones de las piezas, el adhesivo que se emplee tiene que tener un suficiente grado de flexibilidad.

El producto utilizado en estos casos debe ser un mortero cola cementoso mejorado- $\mathrm{C} 2$, con aditivo polimérico a base de látex (S1 para los deformables ó S2 para los muy deformables), con tiempo abierto ampliado-E y deslizamiento reducido-T, tipo C2-TE-S1 o C2-TE-S2 (1).

La técnica de colocación debe garantizar que este adhesivo se aplica a toda la superficie de la pieza, con lo que conseguimos los siguientes efectos:

$1^{0}$. Se permite un mejor reparto de tensiones al asegurar la máxima superficie de contacto entre el soporte y el adhesivo, y entre éste y la baldosa.

$2^{0}$. Se evita la formación de eflorescencias, al impedir el estancamiento del agua de lluvia o de condensación en el trasdós de la baldosa.

$3^{\circ}$. Se mejoran las condiciones de adherencia de las baldosas para soportar en mejores condiciones el efecto de posibles heladas.

Para ello se debe peinar la cola sobre el soporte, con una llana dentada de tamaño de diente adecuado, que asegure un espesor regular y una buena distribución de la cola por toda la superficie.

La colocación con doble encolado (aplicación tanto en el soporte como en la pieza) garantiza la perfecta adhesión en las piezas cerámicas de gran formato (área superior a $2.000 \mathrm{~cm}^{2}$ / pieza) y evita que se formen huecos entre éstas y el soporte (4).

Por último, la adherencia exigida a los adhesivos cementosos (tipo $\mathrm{C}$ ) es de al menos $0,5 \mathrm{~N} / \mathrm{mm}^{2}$ para los adhesivos de fraguado normal y rápido, y 1,0 N/ $\mathrm{mm}^{2}$ para los adhesivos de alta adherencia, como el C2 (1).

\subsubsection{Deformación-cizalladura}

La colocación de una baldosa cerámica en una fachada produce que su peso propio, como carga vertical, genere esfuerzos de cizalladura sobre el adhesivo que lo mantiene contra la pared, y por lo tanto, no se deben descartar estos esfuerzos de cortante como posible causa del desprendimiento de las baldosas cuando éstos se producen.
Porcar (6) menciona que el profesor J.K. Felixberger, director del Departamento de Técnica de Aplicación de la empresa PCI Augsburg Gmbh y profesor de la Universidad Estatal de San Petersburgo aporta una expresión matemática de la tensión máxima de cizalladura a que está sometida una baldosa que es función de:

a) La variación de posición entre el soporte y la baldosa, normalmente como diferencia entre la retracción que ha sufrido el primero y la expansión de ésta última.

b) La rigidez de la baldosa, a través de su módulo elástico.

c) El espesor de la capa de adhesivo.

d) La rigidez del adhesivo, a través de su módulo de cizalladura.

e) Las dimensiones de la baldosa.

En las primeras reuniones del Grupo de Trabajo WG3, de CEN/TC 67 para elaborar el contenido de la norma europea sobre adhesivos EN 12004 se consideró la posibilidad de adoptar un método de ensayo para la medida de la resistencia a la cizalladura de los adhesivos cementosos, descartándose al producirse un desplazamiento muy pequeño, del orden de décimas de milímetro (6).

Finalmente la UNE-EN 12004-2008 (1) en su tabla 1, para los adhesivos cementosos, considera la «deformación transversal» como una característica opcional especial, a establecer conforme a la UNE-EN 12002 (3).

El método de ensayo de la UNE-EN 12002 ha sido cuestionado sobre todo por la dispersión de los resultados que puede llegar a alcanzarse en un mismo laboratorio (hasta un $50 \%)$ y que puede doblarse si se trata de ensayos efectuados con el mismo adhesivo en laboratorios diferentes. También resulta inconveniente la confección de las probetas ya que es prácticamente imposible obtener la especificación de espesor que marca la norma, de 3,0 $\pm 0,1 \mathrm{~mm}$, alcanzándose desviaciones de hasta $0,4 \mathrm{~mm}$ incluso por personas que están habituadas a la confección de probetas (6). Por ello, se deben proponer alternativas a la normativa que desemboquen en una mejor caracterización de la deformabilidad de los adhesivos y materiales de rejuntado cementosos.

Por otra parte, Goldberg propugna desarrollar criterios de ingeniería más objetivos y aceptados para el diseño y la especificación de estos adhesivos, tales como los requisitos del módulo de elasticidad, módulo de cizalladura y resistencia a la cizalladura (7).

Teniendo en cuenta que la deformación transversal o resistencia a la cizalladura no es una característica considerada como «fundamental» para clasificar y evaluar la conformidad de los adhesivos cementosos por la UNE-EN 12004 (1); los problemas de preparación de probetas según la UNE-EN 12002 y la dispersión de resultados que ello origina; y que los adhesivos cementosos modificados con polímeros que proporcionan un módulo de cizalladura equilibrado cuentan con una historia empírica probada de 50 años de aplicación satisfactoria (7), ya que los adhesivos cementosos con un alto contenido en resinas, especialmente frente a la colocación de baldosas de gran tamaño, juegan un papel determinante en la contención de las tensiones de cizalladura (6), se ha desistido de realizar esta evaluación en el aplacado de baldosas cerámicas en servicio. 


\subsubsection{Anclajes mecánicos}

En el caso de los aplacados pegados en fachada, a partir de un cierto tamaño de la baldosa que se pega y en revestimientos verticales exteriores, se debe complementar la fijación con anclajes mecánicos (8) (9) (10) (11).

\subsubsection{Juntas de colocación}

Se denomina «junta de colocación» o «juntas entre baldosas» al espacio que queda entre dos baldosas adyacentes.

La junta de colocación tiene las siguientes funciones técnicas:

$1^{\mathrm{o}}$. Absorber las tensiones de compresión y tracción que se puedan producir en el ámbito del recubrimiento.

$2^{0}$. Posibilitar la difusión de vapor de agua desde los estratos interiores del cerramiento (las baldosas de gres porcelánico, dada su nula porosidad, no permiten la difusión del vapor de agua a su través).

$3^{\mathrm{o}}$. Absorber las desviaciones dimensionales de longitud y anchura de las baldosas, dentro de las tolerancias permitidas a las mismas.

Las recomendaciones en cuanto a las dimensiones mínimas de estas juntas oscilan entre $1,5 \mathrm{~mm}$ y $5 \mathrm{~mm}$, según los autores y la situación del revestimiento, en interior o al exterior, respectivamente (4) (8).

Las juntas de colocación afectan únicamente a las baldosas, y se rellenan bien con una lechada de cemento (sistema tradicional cada vez más en desuso) o con mortero de juntas, morteros industriales de base cementosa pero con aditivos que mejoran su adherencia a los bordes de las baldosas y su elasticidad frente a pequeños movimientos.

\subsubsection{Juntas de movimiento}

La colocación de las baldosas cerámicas requiere de juntas de movimiento o juntas de dilatación, particularmente en exteriores, subdividiendo los grandes paños en recuadros de menores dimensiones, con la finalidad de evitar acumulación de tensiones por dilataciones y contracciones térmicas, o por movimientos del soporte (deformaciones del cerramiento por los empujes del viento o por las flechas de los forjados en que se apoyan, siempre dentro de las tolerancias permitidas a estos elementos constructivos). Las referencias de que se disponen recomiendan las siguientes separaciones para estas juntas de movimiento:

a) Disposición de juntas de dilatación o juntas de movimiento entre paños de $25 \mathrm{~m}^{2}$ a $35 \mathrm{~m}^{2}$ en el exterior (entre $5 \mathrm{~m}$ y 6 m de longitud) (4).

b) Utilización de juntas de movimiento de tal forma que se fraccione el revestimiento en los cantos de los forjados, en los cambios de plano y cada $9 \mathrm{~m}^{2}$, como máximo, con baldosas de formato mayor a $900 \mathrm{~cm}^{2}(30 \mathrm{~cm} \times 30 \mathrm{~cm})$ (12) (9).

Las juntas de dilatación o juntas de movimiento del revestimiento con baldosas cerámicas debe afectar a las baldosas y a su material de agarre al soporte, y tener entre 5 y $10 \mathrm{~mm}$ de ancho, dependiendo del color y exposición a cambios de temperatura, que se rellenará con un material elástico del tipo de un sellador silicónico o un sellante a base de poliuretano (12).

\section{RESULTADOS Y DISCUSIÓN}

\subsection{Comprobación de la capacidad de adherencia y del método de aplicación del material de agarre}

La capacidad de adherencia se ha comprobado sometiendo a tracción mediante un adherómetro digital un total de 60 probetas extraídas in situ. La realización de la prueba se realiza según el siguiente procedimiento:

a) Elección del lugar donde tomar la muestra. Se buscó un reparto uniforme de los testigos ensayados, con la finalidad de hacer al menos dos pruebas en cada uno de los bloques, y que estas pruebas fuesen variadas en cuanto a posición, realizándolas no sólo en las zonas accesibles a ras de suelo, sino también en altura empleando una plataforma elevadora de tijeras diésel para ello.

b) Perforación de la baldosa, hasta llegar al mortero de agarre, con una corona de diamante refrigerada por agua, de $50 \mathrm{~mm}$ de diámetro, que cuenta con una barrena central que hace de guía.

Si bien la norma UNE-EN 1348 establece en el procedimiento de ensayo la preparación en laboratorio de probetas cuadradas de $50 \mathrm{~mm}$ de lado (2), se tuvo en cuenta la dificultad que esto podía suponer, pues habrían de ser talladas, con una radial por ejemplo, sobre un aplacado colocado, suponiendo un mayor riesgo de dispersión en los tamaños y de alteración de la propia probeta, así como una extracción más destructiva para las fachadas de unos bloques de viviendas en uso. Por ello, se optó por la perforación con corona de diamante, método que era menos destructivo para la fachada y más fiable en cuanto supone menor alteración de la muestra.

c) Aplicación del adhesivo que servirá para unir la sufridera metálica a la baldosa. Se ha empleado un adhesivo de doble componente tipo Nural 23. Colocación de la sufridera.

d) Identificación de la sufridera antes de proceder a su extracción, con el número del ensayo y el bloque donde se encuentra.

e) Colocación del perno que servirá de asidero al aparato que tiene que tirar de la sufridera (Figura 1.a).

f) Agarre de la cabeza del perno al adherómetro y aplicación de la fuerza de tracción hasta arrancar el conjunto formado por sufridera-baldosa, con medición directa de la tensión aplicada (Figuras 1.b y 1.c).

g) Observación y registro de la rotura (Figuras 2.a, 2.b y 2.c).

Se han obtenido distintos tipos de rotura tras la aplicación de la fuerza de tracción.

Rotura por adhesión, en la cual la baldosa aparece limpia ya que la fuerza aplicada ha alcanzado la fuerza de adhesión del adhesivo (Figura 2a).

Rotura por cohesión (Figura 2b), aparecen restos de adhesivo adherido a la baldosa ya que la fuerza aplicada ha alcanzado la fuerza de cohesión interna del adhesivo, que es menor que la fuerza de adhesión con la baldosa.

Despegue del adhesivo del ladrillo del cerramiento (Figura 2c), la fuerza de adhesión del adhesivo al ladrillo o al enfoscado es inferior a la fuerza de adhesión a la baldosa y a la fuerza de cohesión interna del adhesivo. 
Dado que el adherómetro empleado nos informa de la tensión en $\mathrm{kg} / \mathrm{cm}^{2}$ que se ha producido en el momento de la rotura de la probeta, y a la vista de los resultados en cuanto a la superficie real de contacto entre la baldosa y el adhesivo, inferior a la teórica, pues se han detectado muchas zonas huecas en el trasdós de la probeta, se ha corregido el valor de la tensión medida con la superficie real de contacto de cada probeta, para obtener el valor real, no el teórico, de la resistencia a la tracción del adhesivo.

Los resultados obtenidos mostraron que el $35 \%$ era inferior a $0,5 \mathrm{~N} / \mathrm{mm}^{2}$, el $17 \%$ estaba entre $0,5 \mathrm{y} 1 \mathrm{~N} / \mathrm{mm}^{2}$ y el $48 \%$ superaba $1 \mathrm{~N} / \mathrm{mm}^{2}$, como puede comprobarse en el gráfico de la Figura 3.

También se pudo comprobar que de las probetas con una resistencia a tracción inferior a $0,5 \mathrm{~N} / \mathrm{mm}^{2}$, el $81 \%$ había falla- do por rotura de adhesión entre la baldosa y el adhesivo (el resto rompió por cohesión del adhesivo). Este tipo de roturas, con resistencias tan bajas, es consecuencia de haber aplicado el adhesivo con el tiempo abierto excedido.

En cuanto a la forma de aplicación del material de agarre, se ha podido comprobar que ha sido mediante pellas de pasta sobre las baldosas, para colocarlas en unas ocasiones directamente sobre el enfoscado de mortero de cemento de la fábrica, y otras mediante un encolado previo del enfoscado, en una variante poco ortodoxa de la técnica de doble encolado. Dicha comprobación se ha efectuado por los siguientes procedimientos:

a) Golpeando las baldosas. Las zonas huecas se han detectado tanto en las esquinas y bordes de las baldosas, como en zonas intermedias de las mismas.

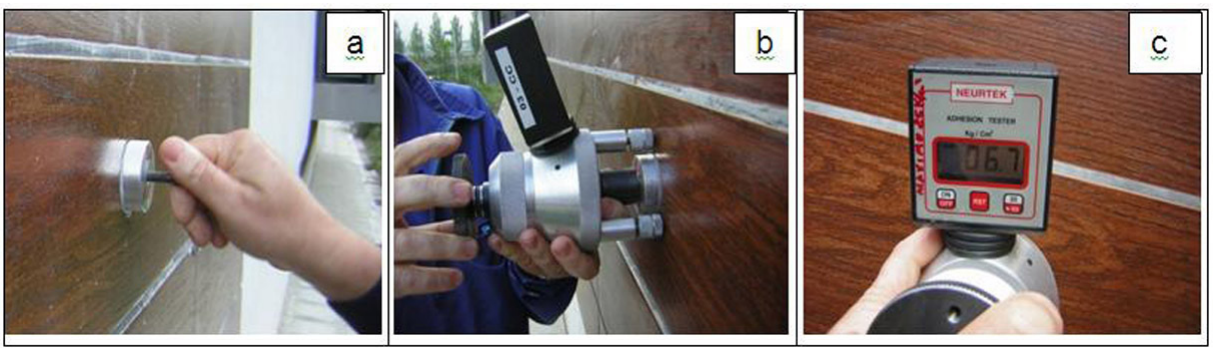

Figura 1. Proceso de obtención de muestras.

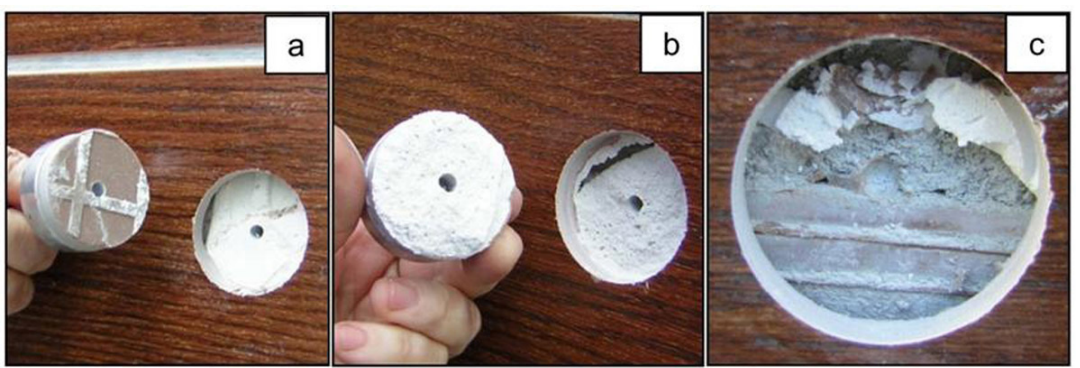

Figura 2. Observación y registro de la rotura.

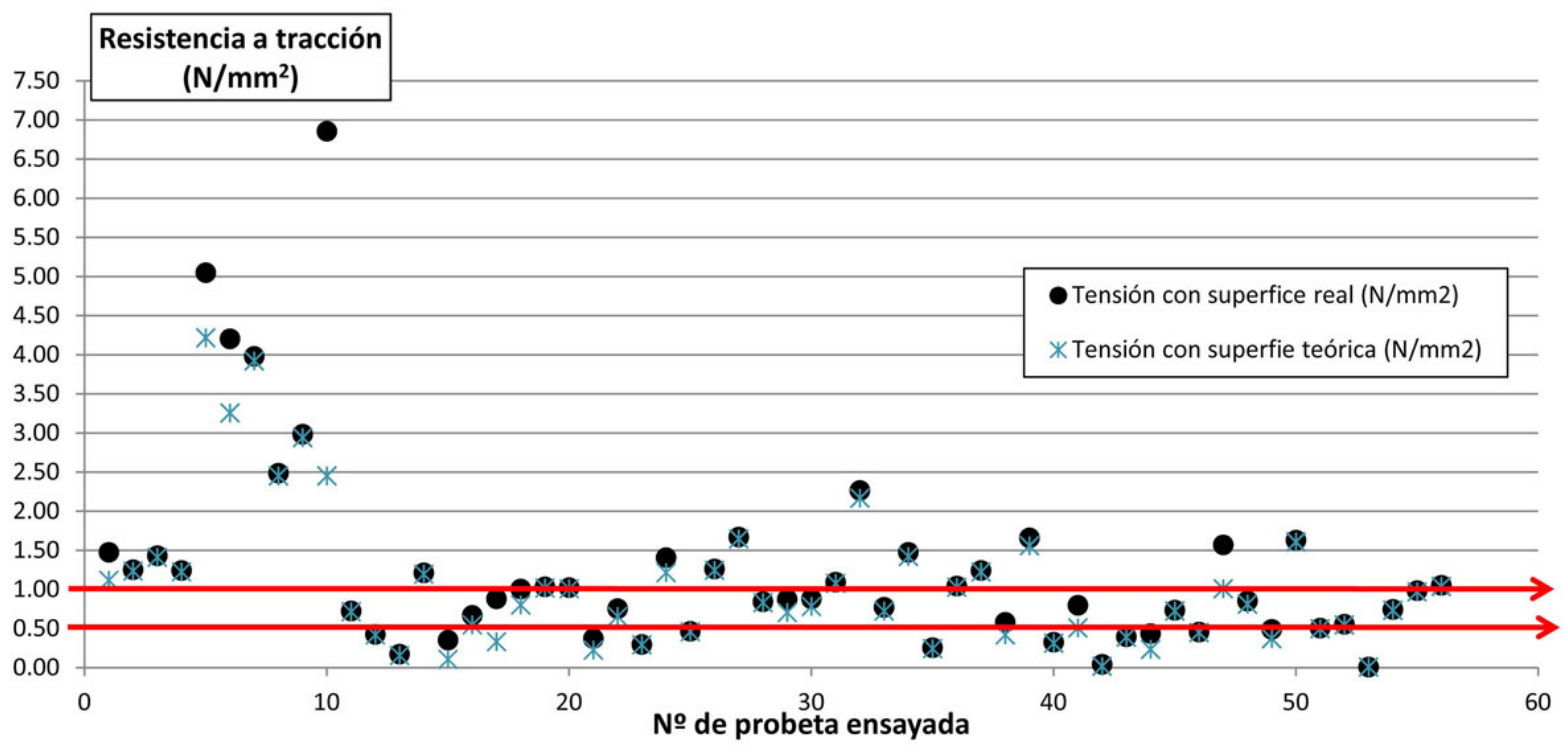

Figura 3. Gráfico que muestra la resistencia a tracción, tanto teórica como real de cada una de las probetas ensayadas. 
b) Analizando las fotografías de los desprendimientos de las baldosas que se tomaron antes de que fuesen reparados por el constructor, donde se podía apreciar con claridad la colocación por pellas.

c) Analizando el trasdós de las baldosas tras la extracción de las placas para la determinación de la fuerza de adherencia (Figuras 4.a, 4.b y 4.c).

d) Analizando los orificios que quedaban tras el desmontado de las chapas de acero inoxidable vistas que presionan las baldosas, en las pruebas de detección de los anclajes ocultos.

La colocación del adhesivo por pelladas, dispuestas en las zonas centrales de las baldosas y no en sus bordes, dejan los bordes huecos en su trasdós. Al ser el borde de la baldosa el lugar de sujeción del anclaje mecánico, que va atornillado al soporte, dicho anclaje está en el aire, al carecer de relleno con adhesivo, lo que anula su posible eficacia en caso de fallo del adhesivo, pues no es un anclaje firme (Figuras 5 y 6 ).

Además de la pérdida de estabilidad que esta deficiente colocación de los anclajes produce por colocarse el tornillo empotrado solo por su punta, los huecos tras las baldosas, al permitir la escorrentía del agua de lluvia y condensación por su trasdós, provocan un proceso de hidratación de los silicatos, de carbonatación, con posible transformación parcial a bicarbonato cálcico (solubilidad de $745 \mathrm{~g} / \mathrm{l}$, frente a 1,8 g/l de la portlandita) o suspensión de polvo acumulado que llevan a la aparición de chorreones blanquecinos sobre la superficie exterior del aplacado.

\subsection{Comprobación de la existencia de los anclajes mecánicos de las baldosas}

La comprobación de la existencia de los anclajes mecánicos ocultos se ha realizado con un detector de metales. Las baldosas vienen mecanizadas de fábrica con una ranura en el extremo del canto que permite la colocación de estos anclajes ocultos. Para intentar evitar que las baldosas se siguieran cayendo, el constructor había colocado unos anclajes mecánicos vistos compuestos por una chapa de acero inoxidable de $50 \times 30 \mathrm{~mm}$, con juntas de goma en la superficie de contacto con las baldosas, sujetas por la presión de un tornillo central colocado en el cruce de las juntas de mortero entre baldosas. Estas chapas se desmontaron para evitar que causaran interferencias en la detección de los anclajes ocultos, dada la proximidad entre unos y otros (ambos muy próximos a las esquinas).

Se pudo comprobar que no existían anclajes ocultos en las juntas horizontales del arranque de las fachadas, ni en los bordes donde fue necesario cortar las baldosas: encuentros con alféizares y mochetas de ventanas o finales de paños de fachadas con baldosas cortadas verticalmente. Esta ausencia
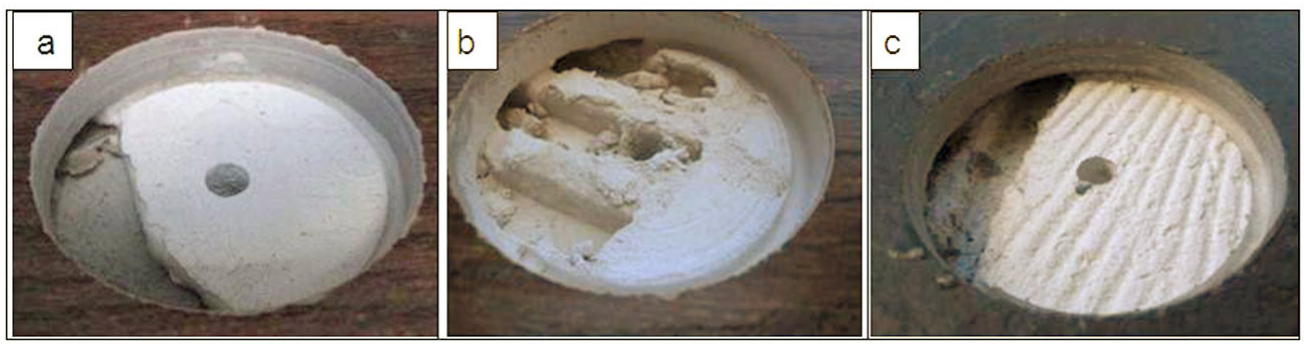

Figura 4. Análisis del trasdós de la baldosa, para comprobar la forma de colocación del adhesivo.
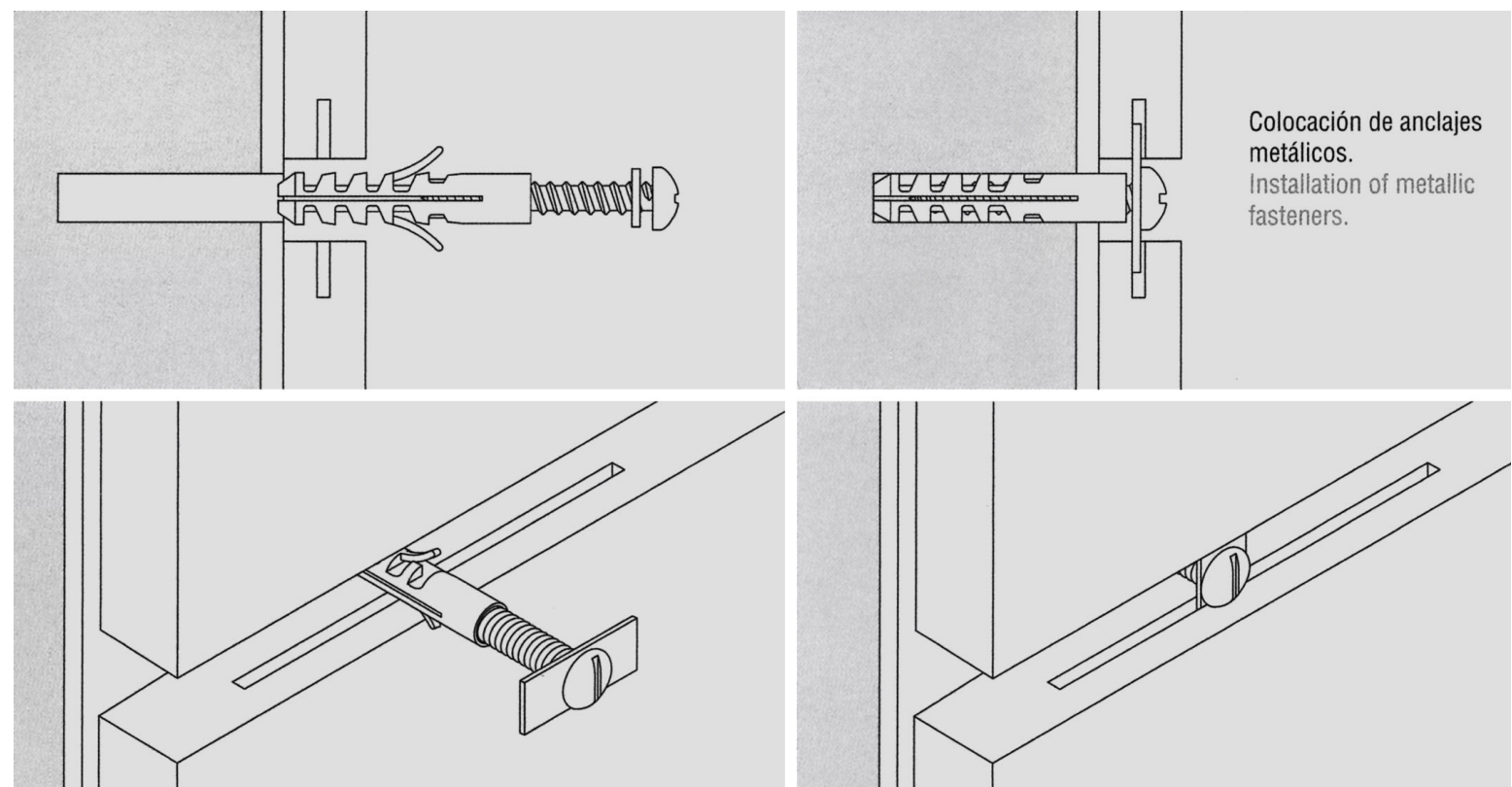

Figura 5. Colocación de anclaje oculto: la baldosa tiene apoyo en el adhesivo colocado en su trasdós, el anclaje está perfectamente empotrado, pudiendo garantizar así su estabilidad (9). 


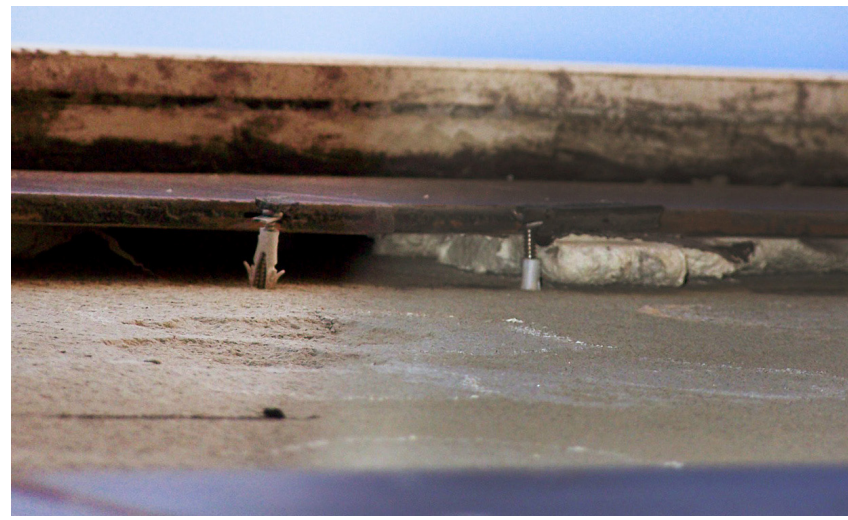

Figura 6. Canto de una baldosa pegada por pelladas. El tornillo de fijación del anclaje está empotrado solo por la punta, por lo que el anclaje pierde estabilidad y capacidad de soportar la baldosa. Fotografía tomada in situ, tras el desprendimiento de una baldosa.

de anclajes en zonas donde las baldosas son más vulnerables por tratarse de piezas cortadas, favorece la caída de las baldosas en caso de pérdida de adhesión con su soporte.

\subsection{Análisis de las juntas de colocación entre baldosas}

El análisis se efectuó con una inspección visual, midiendo la distancia entre dos bordes de baldosas contiguas. Como las baldosas no eran rectificadas y tenían los bordes ligeramente achaflanados, fue necesario retirar con una espátula el mortero superficial del fraguado entre baldosas para poder acceder al borde exterior del chaflán, algo más rehundido respecto de la superficie de la baldosa.

Se observó que tanto las baldosas de $50 \times 33 \mathrm{~cm}$ como las $66 \times 37 \mathrm{~cm}$ estaban colocadas con juntas verticales de $1 \mathrm{~mm}$ de grosor máximo, detectándose numerosas piezas colocadas a hueso, con los bordes en contacto entre dos baldosas contiguas. La falta de dimensión suficiente en las juntas entre baldosas hace que cualquier aumento de dimensión de las baldosas (expansión por dilatación térmica o por humedad) no tenga capacidad de ser absorbido por el espacio entre ellas y se traduzca en tensiones que provoquen su desprendimiento.

En las juntas verticales se observó cierta homegeneidad, con grosores en torno a los $8 \mathrm{~mm}$.

\subsection{Análisis de las juntas de movimiento del revestimiento}

El análisis se efectuó con una inspección visual y una comprobación táctil pasando el canto de una espátula de acero inoxidable de $1 \mathrm{~mm}$ de espesor por la junta, para detectar posibles sellados con material elástico. Se revisaron juntas verticales en paños de longitud en horizontal superior a $5 \mathrm{~m}$, y juntas horizontales en toda la altura del edificio, ayudados de plataformas móviles de tijeras.

Finalmente no se detectaron juntas de movimiento.

\section{CONCLUSIONES}

De las comprobaciones efectuadas se pueden determinar las causas de los desprendimientos de las baldosas, que en el caso que nos ocupa son varias:
1) El adhesivo utilizado se aplicó en muchas ocasiones con el tiempo abierto excedido, El $52 \%$ de las resistencias a tracción obtenidas en las pruebas resultaron por debajo de $1 \mathrm{~N} / \mathrm{mm}^{2}$, mientras que el $48 \%$ restante sí obtuvieron las resistencias esperadas.

2) El método de colocación empleado fue diverso e inadecuado. En general se puede decir que se colocó mediante pellas. Esta aplicación desordenada y que no cubría la superficie completa de las baldosas ha disminuido su capacidad de adhesión al soporte, en un material donde este aspecto hay que cuidarlo especialmente por su baja absorción de agua, al ser un material muy compacto y requerir por lo tanto fijación química.

3) La colocación mediante pellas ha dejado hueco el trasdós de los bordes de las baldosas, por lo que los anclajes mecánicos tenían solo la punta del tornillo de fijación empotrada, no pudiendo cumplir su misión de sujetar las baldosas en caso de fallo del adhesivo, al quedar el anclaje inestable.

4) La colocación de las baldosas sin juntas de colocación en horizontal y sin juntas de dilatación en vertical, donde la longitud del paño supera los cinco metros (edificios entre cuatro y seis plantas de altura), favorece el desprendimiento de las baldosas por las dilataciones térmicas que experimentan sin que cuenten con estas juntas para absorber los movimientos.

5) Los huecos existentes en el trasdós de las baldosas favorecen la escorrentía del agua, que puede penetrar a través de sus juntas, arrastrando polvo y restos de adhesivo que manchan la superficie exterior del aplacado.

Como conclusión final de este estudio se propone un procedimiento de diagnóstico para determinar las causas del desprendimiento de las baldosas de un aplacado cerámico en servicio, llevando a cabo las siguientes actuaciones:

a) Determinación de los parámetros de referencia a adoptar, en función del material y tamaño de las baldosas y de su situación en el edificio.

b) Comprobación de que las especificaciones contenidas en el proyecto de ejecución de las obras son adecuadas para el sistema constructivo de aplacado elegido.

c) Comprobación de la capacidad de adherencia del adhesivo empleado para la colocación de las baldosas. Ensayos in situ mediante adherómetro digital.

d) Comprobación del sistema de aplicación del adhesivo: totalmente extendido por la superficie o por pellas; simple o doble encolado; y tiempo abierto. Análisis de resultados mediante la observación de las muestras extraídas con el adherómetro.

e) Comprobación de la existencia de anclajes ocultos en caso de ser necesarios (por revestimiento en altura o por piezas de gran formato) mediante pruebas no destructivas, con un detector de metales.

f) Verificación de la adecuación de las juntas de colocación.

g) Verificación de la adecuación de las juntas de movimiento necesarias.

h) Verificación de la resistencia a cizalladura del adhesivo. Si el resto de pruebas no determina con claridad las causas de los desprendimientos de las baldosas, será necesario extraer muestras y someterlas al ensayo de rotura por cizalladura. Esta prueba tendrá carácter residual por la dificultad de preparación de las probetas y la fácil dispersión que pueden sufrir los resultados. 


\section{REFERENCIAS}

(1) AENOR. (2008). UNE-EN 12004:20o8 - Adhesivos para baldosas cerámicas. Requisitos, evaluación de la conformidad, clasificación y designación. Asociación Española de Normalización (AENOR).

(2) AENOR. (2008). UNE-EN 1348:2008 - Adhesivos para baldosas cerámicas. Determinación de la resistencia a la tracción de los adhesivos cementosos. Asociación Española de Normalización (AENOR).

(3) AENOR. (2009). UNE-EN 12002:2009 - Adhesivos para baldosas cerámicas. Determinación de la deformación transversal de adhesivos y materiales de rejuntado cementosos. Asociación Española de Normalización (AENOR).

(4) Ascer (Asociación Española de Fabricantes de Azulejos, Pavimentos y Baldosas Cerámicas). (2006). Guía de la Baldosa Cerámica. Valencia: Institut Valenciá de la Edificació.

(5) AENOR. (2007). UNE-EN 14411:2007 - Baldosas cerámicas. Definiciones, clasificación, características y marcado. Asociación Española de Normalización (AENOR).

(6) Porcar, J.L. (2006). Adherencia y deformabilidad de los adhesivos y materiales de rejuntado cementosos para la colocación de baldosas cerámicas y otros aplacados rígidos modulares. En Qualicer'o6 - IX Congreso mundial de la calidad del azulejo y pavimento cerámico. Castellón.

(7) Goldberg, R.P. (2010). La importancia de las características de resistencia a la cizalladura de los adhesivos cementosos modificados con polímeros. En Qualicer'1o - XI Congreso mundial de la calidad del azulejo y del pavimento cerámico. Castellón.

(8) Departamento Técnico de Anfapa (Asociación Nacional de Fabricantes de Morteros Industriales). (2009). Informe: claves para una correcta colocación de cerámica como revestimiento en fachadas. CIC Arquitectura y Construcción, (467): 36-40,

(9) Butech-Grupo Porcelanosa. (2004). Sistemas constructivos de fachada técnica. Alicante: Grupo Porcelanosa.

(10) Weber. (2009). Colocación y rejuntado de cerámica. ¿Cómo colocar cerámica en fachadas?. En La Guía Weber 2010 (pp. 32-33). Barcelona: Saint Gobain Weber Cemarksa.

(11) Ministerio de la Vivienda. (1973, 16 de junio). NTE-RPC/1973 Norma Tecnológica Española. Revestimientos de Paramentos Chapados. Boletín Oficial del Estado, ${ }^{0}$ 144. Gobierno de España.

(12) Porcar-Ramos, J.L. (2008). Adhesivos y materiales de rejuntado. Colocación de baldosas cerámicas y otros recubrimientos rígidos modulares ( $1^{\mathrm{a}}$ ed.). Madrid: ANFAPA. 